\title{
Research Progress of Graphene Composites
}

\author{
Yuan Gao, Jingwen Wang, Jianwei Li \\ China University of Petroleum, Beijing, 102200
}

\begin{abstract}
Graphene has become a hot topic in the field of materials because of its excellent properties and unique two-dimensional structure. In this paper, the preparation methods of graphene are reviewed and the advantages and disadvantages of each method are analyzed. The mechanical, optical, electrical and thermal properties of graphene are briefly introduced. In this paper, the preparation and application of graphene-based polymer composites and graphene-based inorganic nanocomposites are described in detail. The effects of graphene-based composites and graphene-based composites are discussed in detail.
\end{abstract}

Keywords Graphene, Materials Science, Composites

\section{Introduction}

Preparation of graphene From the earliest mechanical stripping method began to develop a variety of preparation methods, such as: crystal epitaxial growth method, chemical vapor deposition, liquid direct stripping method and high temperature deoxidation and chemical reduction method. Researchers in China earlier carried out the preparation of graphene research. Chemical vapor deposition is a common method for preparing large area graphene. At present, hydrocarbon gas (such as $\mathrm{CH} 4, \mathrm{C} 2 \mathrm{H} 2, \mathrm{C} 2 \mathrm{H} 4$, etc.) is used as a carbon source for the precursors. Solid carbon aggregates can also be used as carbon sources. For example, Sun et al. Deposited the polymer thin films onto metal catalyst substrates using chemical vapor deposition, the preparation of high-quality controllable layer of graphene. Compared with the chemical vapor deposition method, plasma enhanced chemical vapor deposition can be in a lower deposition temperature and shorter reaction time to prepare a single graphene. In addition, crystal epitaxial growth method by heating single-crystal $6 \mathrm{H}-\mathrm{SiC}$ removal of $\mathrm{Si}$, 
resulting in $\mathrm{SiC}$ epitaxial growth of graphene. However, the surface of the $\mathrm{SiC}$ crystal will be reconfigured during the process of high temperature, which makes the surface structure more complex, so it is difficult to obtain graphene with large area and uniform thickness. The solvothermal method has been paid more and more attention due to the high quality of graphene prepared under the high temperature and high pressure closed system. Compared with other methods, graphene nanobelts with defect-free structure and definite structure can be prepared by organic synthesis method. Unlike the bottom-up synthesis described above, the top-down approach increases the yield of graphene and is easy to prepare. The subsequent development of solvent stripping method than the redox method toxicity, and will not destroy the structure of graphene. In addition to the chemical reduction method, but also by electrochemical methods to reduce the graphite oxide graphene, but the preparation of graphene obtained in the $\mathrm{C}$ and $\mathrm{O}$ atoms ratio is low. In addition, the microwave method was also used to prepare graphene, such as Chen et al. First, oxidized graphene (GO) was dispersed into a mixed solvent of NN-dimethylacetamide and water (DMAc / H2O), and then the mixed reaction solution was subjected to the thermal conductivity of the graphene was 104 times higher than that of the graphene oxide. Lu Yan of Beijing University of Science and Technology used graphene with an open mesopore structure with a specific surface area of $77.8 \mathrm{~m} 2 / \mathrm{g}$ and a mesoporosity of $74.7 \%$, which can be used as an electrode material.

\section{Graphene Excellent Performance}

One of the most important properties of graphene is its unique carrier characteristics and quality-free Dirac fermion properties. The valence band and the conduction band of graphene overlap at the Fermi level and are twodimensional semiconductors with zero energy gap. The carrier can not move through the submicron distance by scattering. It is the material with the smallest resistivity. The electron mobility of the graphene can be more than $15000 \mathrm{~cm} 2 /$ $(\mathrm{V} \cdot \mathrm{s})$ at room temperature, while when the carrier density is less than $5 \times$ $109 \mathrm{~cm}-2$, the graphene Was first found to be close to $200000 \mathrm{~cm} 2 /(\mathrm{V} \cdot \mathrm{s})$. The carrier mobility in monolayer graphene is almost unaffected by chemical doping and temperature. In addition, the half-integer quantum Hall effect of electron carriers and hole carriers in graphene can be changed by the electric field to change the chemical potential., While Novoselov et al. Observed this quantum Hall effect of graphene at room temperature. Burghard et al. Found that the conductivity of the chemically reduced multilayer graphene oxide sheet is between 0.05 and $2 \mathrm{~S} / \mathrm{cm}$, and the field-effect mobility at room temperature is 2 to $200 \mathrm{~cm} 2 /(\mathrm{V} \bullet \mathrm{s})$.

The white light absorptivity of monolayer suspended graphene is $2.3 \%$, and the absorption rate increases linearly with the number of layers. Gusynin et al. Found that the transparency of graphene only depends on its fine structure constant. Dawlaty of Cornell University studied the carrier dynamics and relative relaxation time of graphene grown on $\mathrm{SiC}$ using ultrafast optical pumping-probe 
spectroscopy. They found a fast relaxation transient: 70 to 120 fs followed by a slow relaxation process of 0.4 to $1.7 \mathrm{ps,} \mathrm{respectively,} \mathrm{with} \mathrm{the} \mathrm{graphene} \mathrm{carrier-}$ carrier in-band scanning process And the carrier - phonon interband scanning process. Wang et al. Found that inter-band and optical transitions of multilayer graphene can be controlled by electronic gating techniques using infrared spectroscopy.

The thermal conductivity of monolayer suspended graphene can reach 3000$5000 \mathrm{~W} /(\mathrm{m} \cdot \mathrm{K})$ at room temperature, which can be used to solve the heat dissipation problem in nanoelectronics, even though the graphene flake size is different. Seol et al. Demonstrated that graphene grown on amorphous $\mathrm{SiO} 2$ can still achieve a thermal conductivity of $600 \mathrm{~W} /(\mathrm{m} \bullet \mathrm{K})$ and a decrease in thermal conductivity due to the presence of The dissipation of the phonon through the graphene-silicon interface, and the strong interfacial scattering, the thermal conductivity of graphene is still twice that of copper and 50 times that of silicon, which is now widely used in the electronics field.

Lee et al. Tested the mechanical properties of the independently supported multilayer graphene using atomic force microscopy (AFM) nanoindentation techniques. They found that the graphene had a fracture strength of $42 \mathrm{~N} / \mathrm{m}$ and the Young's modulus of the ultra-narrow graphene ribbon was about $7 \mathrm{TPa}$, is currently known as the most solid material. In addition, Hao et al. Used quantum mechanics to study the fracture mechanism of graphene graphene and graphene. They found that both graphene models started from the outermost carbon atom layer. And Frank and other experiments by using the cantilever found that single graphene can withstand different angles of axial pressure. After repeated folding of the graphene sheets, the zigzag lines appear in the same position, which is due to kinks and defects already present on the sheet.

\section{Graphene-Inorganic Composites}

There are two main ways to prepare graphene-inorganic composites: firstly, oxidized graphite is compounded with other materials, then graphite oxide is reduced to obtain graphene composites; secondly, graphene is directly compounded with other materials. No matter what kind of compound method is adopted, nano-inorganic materials are dispersed on the graphene sheets and graphene-inorganic nanocomposites are obtained by the combination of graphene or graphene graphene by electrostatic attraction.

When the graphene is compounded with a metal compound, the metal compound commonly used includes metal oxides, metal sulfides, metal hydroxides and the like. Graphene has a large specific surface area and good electrical conductivity, but in practical applications, due to the accumulation of graphene layers to double the effective area of the lower, resulting in ultra-low energy density of the capacitor, the catalytic activity decreased. The preparation of graphene metal compound composites by different methods can effectively increase the electric double layer of graphene, so as to improve the specific capacitance and charge-discharge efficiency of the capacitor. 
The graphene can be compounded with other inorganic materials such as metal oxides and carbon nanotubes. Single graphene has a high specific surface area, good electrical conductivity, in the actual study found that graphene tends to produce accumulation, resulting in lower specific surface area. When graphene and other inorganic composites are prepared by different methods, the intercalation of the inorganic substance into the graphene layer plays a role of skeleton support and increases the specific surface area of graphene, which provides more holes for the embedding and emigration of ions, Thus improving the conductivity of composite materials and other properties.

\section{Graphene-Polymer Composites}

Epoxy resins, polyurethanes, polystyrenes, polyvinyl alcohols, polyethylenes, polypropylenes, conductive polymers, and the like are common polymers when graphene is compounded with a polymer. These polymers are easily covalently bonded to the graphene surface or edge regions when they are combined with graphene, breaking the Van der Waals force between the graphene sheets. The modified graphene composites have good mechanical properties, electrical conductivity, electromagnetic wave absorption Rate and so on.

WU Chunxia Nano-graphene-carbon nanofibers were prepared by high pressure controlled physical filtration. This nano-paper can effectively reduce the surface of the electromagnetic wave reflection, improve the absorption efficiency of electromagnetic waves. At the same time, with the increase of graphene content, the conductivity of nano-paper is improved. The epoxy resin into the nano-paper gap, so that nano-paper and epoxy resin effectively together to complete the force, heat transfer, can make the electromagnetic absorption efficiency as high as $97.1 \%$.

Raghu and other mixed polyols, chain extenders and polyisocyanate in situ polymerization of polyurethane matrix, with graphene as filler to the matrix to get graphene-polyurethane composite materials. For a two-phase composite of the same hard segment (short segment of hard segment, which is a long segment of the polymer that forms plastic properties at room temperature in a multi-block copolymer), as the graphene content increases, Its conductivity increases rapidly until it reaches a certain platform. When the content of graphene is the same, the conductivity of the composites with low hard - segment content is higher than that of the hard - segment composites. Compared with the pure polyurethane, the hardness value of the graphene-urethane composite will be improved, and the hardness of graphene on the low hard segment content is higher than that of the high hard segment.

Used graphene as matrix and ammonium persulfate as oxidant to synthesize graphene-polyaniline composites in an acidic system by in-situ polymerization. It was found that the interaction between graphene and polyaniline decreased with the increase of polymerization time, and the adsorption capacity of graphene to aniline decreased with the addition of other reagents. The interface of the graphene-polyaniline composite with $\mathrm{CdS}$ was remarkably improved, and the 
particle size of CdS also had some influence on the interface quality. The composite electrode prepared by the ultra-graphene graphene electrode than the performance significantly improved. Wang Hongzhi and other electrochemical methods to aniline and oxidized graphene as raw materials, polyaniline evenly distributed to the graphene layer, the synthesis of graphene-polyaniline composite materials. The composite has a current density of $500 \mathrm{~mA} / \mathrm{g}$, a specific capacitance of $325 \mathrm{~F} / \mathrm{g}$, a current density of $1000 \mathrm{~mA} / \mathrm{g}$ and a specific capacitance of $315 \mathrm{~F} / \mathrm{g}$. After not less than 1000 cycles of charge and discharge, the ratio of capacitance retention is still as high as $90 \%$. And the discharge efficiency of the composite material is extremely high, and the electrolyte ions in the electrode are easy to diffuse and migrate.

\section{Conclusion}

Graphene is a research hotspot because of its excellent performance. The preparation process of the graphene has been developed from the earliest mechanical stripping methods such as chemical vapor deposition, oxidation reduction, liquid phase stripping, crystal epitaxial growth and so on. Preparation methods, but the preparation of large quantities of low-cost high-quality graphene graphene preparation is still a focus of future research. At present, the research of graphene composites is mainly focused on graphene polymer materials and graphene surface-supported inorganic nanoparticles and their applications in catalysis, biosensor, spectroscopy and so on. The research on graphene metal matrix composites the excellent enhancement effect of graphene and its unique interface with the metal matrix will make the composites become the research hotspots of future graphene composites.

\section{References}

[1] Sun Fengqiang, Chen Ying. Structured graphene and its composites [J]. Journal of South China Normal University, 2016(05)

[2] Chen Ying, Zhao Yu, Han Xingyue. Study on the structure and electrical conductivity of $\mathrm{TiO} 2 /$ graphene composites $[\mathrm{J}]$. Bulletin of the Chinese Ceramic Society, 2014(01)

[3] Zhang Xiaohua, Zhou Shijing. Preparation of ultrahigh molecular weight polyethylene / graphene nanocomposite and its thermal properties [J]. Materials Science and Technology, 2016(05)

[4] Liu Chongchong, Xu Hui, Zhang Yahui, Ji Xiucui, Lan Wei, Wang Lei. Study on the Antibacterial Properties of Graphene Oxide and Its Metal Composite Materials [J].Journal of Ludong University, 2012 (06)

[5] Xiao Fafa, Chen Kaikai. Advances in the study of graphene adsorption and separation functional materials [J]. Journal of Textile Research, 2016 (10) 